\title{
Comparative transcriptome analysis of the Asteraceae halophyte Karelinia caspica under salt stress
}

\author{
Xia Zhang ${ }^{*}$, Maoseng Liao, Dan Chang and Fuchun Zhang ${ }^{*}$
}

\begin{abstract}
Background: Much attention has been given to the potential of halophytes as sources of tolerance traits for introduction into cereals. However, a great deal remains unknown about the diverse mechanisms employed by halophytes to cope with salinity. To characterize salt tolerance mechanisms underlying Karelinia caspica, an Asteraceae halophyte, we performed Large-scale transcriptomic analysis using a high-throughput Illumina sequencing platform. Comparative gene expression analysis was performed to correlate the effects of salt stress and ABA regulation at the molecular level.
\end{abstract}

Results: Total sequence reads generated by pyrosequencing were assembled into 287,185 non-redundant transcripts with an average length of $652 \mathrm{bp}$. Using the BLAST function in the Swiss-Prot, NCBI nr, GO, KEGG, and KOG databases, a total of 216,416 coding sequences associated with known proteins were annotated. Among these, 35,533 unigenes were classified into 69 gene ontology categories, and 18,378 unigenes were classified into 202 known pathways. Based on the fold changes observed when comparing the salt stress and control samples, 60,127 unigenes were differentially expressed, with 38,122 and 22,005 up- and down-regulated, respectively. Several of the differentially expressed genes are known to be involved in the signaling pathway of the plant hormone ABA, including ABA metabolism, transport, and sensing as well as the ABA signaling cascade.

Conclusions: Transcriptome profiling of $K$. caspica contribute to a comprehensive understanding of $K$. caspica at the molecular level. Moreover, the global survey of differentially expressed genes in this species under salt stress and analyses of the effects of salt stress and ABA regulation will contribute to the identification and characterization of genes and molecular mechanisms underlying salt stress responses in Asteraceae plants.

\section{Background}

Salinity is a major environmental factor limiting plant growth and productivity. Thus, salt-tolerant halophytes serve as an excellent resource for the identification of desirable traits and the subsequent development of new crop systems [1]. Understanding the salt tolerance mechanisms in such plants represents an important step towards generating crop varieties capable of coping with environmental stresses. Towards this end, there is still much to learn about the diverse mechanisms employed by halophytes to cope with salinity stress.

\footnotetext{
*Correspondence: xia.zhangxjkt@gmail.com; zfcxju@gmail.com Xinjiang Key Laboratory of Biological Resources and Genetic Engineering, College of Life Science and Technology, Xinjiang University, 14 Sheng li
} Road, Urumqi 830046, China
Karelinia caspica, an herbaceous Asteraceae perennial that grows in saline deserts and swamps [2], has broadspectrum resistance to pests and is also tolerant to salinity, drought, low temperatures, and high temperatures [3]. Due to its extreme desalination capacity, $K$. caspica is viewed as a good pioneer plant for the improvement of saline soil [4]. As a secretohalophyte, $K$. caspica actively absorbs and discharges salt through special glands and salt holes on the leaf surface [2,3]. At the same time, salt stress promotes $K$. caspica succulence [2], which is typical of euhalophytes. Considering these mechanisms in aggregate, the salt tolerance of $K$. caspica as intermediate between that of euhalophytes and recretohalophytes is worth examining.

For most non-model organisms, the lack of whole-genome sequencing data continues to represent a major hurdle for research, as genome sequencing is still largely impractical for 
most eukaryotes and cannot be finished in a short timeframe. In contrast, high-throughput transcriptome analysis can be performed whether or not genomic sequences for the organism of interest are available [5] and thus represents a feasible approach for organisms that have not been sequenced. To date, transcriptome analysis has been employed in a wide range of eukaryotes including the following: yeast [6], mice [7], humans [8], Arabidopsis [9], Caenorhabditis elegans [10], rice [11], and Vitis vinifera [12].

In addition to regulating diverse aspects of plant growth and development, abscisic acid (ABA) is also required for plant resistance to drought and salt stress [13-15]. Numerous studies have aimed to elaborate the cellular and molecular responses of plants to $\mathrm{ABA}$, including those related to ABA sensing, signaling, metabolism, and transport.

To globally survey the salinity-induced ABA responses in $K$. caspica, RNA-Seq technology was used. The transcription profiles of control and salt-stressed plants were compared, and dynamic changes in the transcriptome were analyzed. The goal of the present work was to elucidate physiological processes, including those involved in the ABA regulatory network, that are induced by salt stress in the halophyte $K$. caspica at the transcriptomic level.

\section{Methods}

\section{Plant materials}

The $K$. caspica seeds used in this study were obtained from saline land (Wujiaqu, Xinjiang, China). Specific permission was not required for this collection activity at these locations, which are considered wastelands, and the field studies did not involve endangered or protected species. Eight-week-old seedlings germinated in perlite: vermiculite substrate (3:1) supplemented with $300 \mathrm{mM}$ $\mathrm{NaCl}$ at $25^{\circ} \mathrm{C}$ and grown under a 16-hour photoperiod were used for the expression analysis. The treated and nontreated groups were sampled at 3, 6, 12, 24, 36, and 48 hours. After washing with deionized distilled water, the material from the different time points was combined to generate the final samples used for cDNA library preparation.

\section{Preparation of cDNA Libraries for RNA-Seq}

For each treatment (i.e., the control and salt stress treatments), approximately $100 \mathrm{~g}$ fresh material was used for RNA preparation. Total RNA was extracted using the RNAqueous Kit [16] then subsequently treated with RNase-free DNase I (QIAGEN \#79254) to remove residual genomic DNA. For each treatment, mRNAs were treated with Truseq RNA sample preparation kit (Illumina-15026495, USA), firstly purified from the $20 \mathrm{mg}$ total RNA using oligo (dT) magnetic beads and fragmented using fragmentation buffer. Cleaved short RNA fragments were used for first-strand cDNA synthesis using reverse transcriptase and hexamer primer, followed by second strand cDNA synthesis using DNA polymerase I and RNase $\mathrm{H}$. Following a quality check using an Agilent 2100 Bioanalyzer, the cDNA libraries were used for sequence analysis using the Illumina HiSeq TM 2000 system.

\section{Transcriptome analysis}

Raw sequencing data were deposited in the DDBJ/EMBL/ GenBank database (accession number GANI00000000). After the adapter sequences were trimmed from the raw reads, empty reads and reads containing unknown nucleotides $(\mathrm{Ns})>5$ were removed. The remaining clean reads were de novo assembled into unigenes using Trinity software (trinityrnaseq_r2012-06-08 edition). The paired-end method was then used to acquire a single set of nonredundant unigenes. Glimmer 3.02 and EMBOSS_6.3 software were used to analyze the coding sequences (CDS) of the unigenes. All non-redundant unigenes were used to perform BLAST searches and to obtain annotation information in the following databases: NCBI nr, Swiss-Prot, Kyoto Encyclopedia of Genes and Genomes (KEGG), and Cluster of Orthologous Groups (KOG). WEGO [17] was used to classify GO function, which was analysis using the Blast2go program [18].

\section{Identification of differentially expressed genes}

RPKM (reads per kilobase per million reads) values were used to evaluate expression and to quantify transcript levels [19], and differentially expressed unigenes [20] were identified by fold change values and fisher test. In the present study, the fold.change $(A B)$ value $\geq 1$ were choosen was used to as the threshold to determine significant differences in gene expression. For the pathway enrichment analysis, all differentially expressed unigenes were mapped to terms in the KEGG database. A search was then performed for significantly enriched KEGG terms compared to the whole transcriptome background.

\section{Quantitative RT-PCR (qRT-PCR) analysis}

For qRT-PCR, total RNA was isolated from $200 \mathrm{mg}$ frozen materials as described above. Three biological replicates were performed for both the control and salt stress-treated seedlings. For each qRT-PCR reaction, 100 ng of RNA that had been treated to remove genomic DNA was used as template for cDNA synthesis. Additional file 1: Table S1 lists the sequences of the primer pairs used for qRT-PCR. The relative transcript levels of selected genes were quantified using Platinum ${ }^{\circledR}$ SYBR $^{\circledR}$ Green qPCR SuperMix-UDG (Invitrogen, CA, USA). The reactions were performed using the 7500 Real-Time PCR system and software (Applied Biosystems, CA, USA). KcACTIN, which was constant under the tested conditions, was included for normalization in each qRT-PCR run. Average expression ratios (PQ/C) were calculated using the $\triangle \Delta C T$ method, and log2 fold-change values were used. 


\section{Results and discussion}

\section{Raw reads processing and assembly}

To obtain transcript information for $K$. caspica, two cDNA libraries were prepared for the control and salt stress-treated plants then sequenced using the Illumina sequencing platform. The raw reads were transformed by base calling from the image data output from sequencing. After trimming the adapter sequences and removing sequences with unknown or low quality bases, approximately 50.35 and 51.58 million clean reads were obtained for the control and salt stress samples, respectively. Trinity software (trinityrnaseq_r2012-06-08) and the pairedend method were used for de novo assembly of the clean reads into contigs. A total of 287,185 transcripts with different lengths were generated from both of the treatments (Additional file 2: Table S2), which provided abundant information for subsequent analysis of salt stress-associated genes in K. caspica.

\section{Functional annotation and GO assignments of the assembled transcripts}

The unigene sets obtained from the $K$. caspica transcriptome data were annotated based on protein sequence homology. First, we used glimmer3.02 and EMBOSS_6.3.1 software to transform all transcripts with sequences longer than 100 bp into reliable coding sequences (CDS). The 320,260 CDS produced were used for further annotation with the NCBI nr, Swiss-Prot, TrEMBL, CDD, pfam, and KOG databases. From this analysis, 216,415 unigenes were identified that exhibited high sequence similarity with known gene sequences (Additional file 3: Table S3 and Additional file 4: Table S4). Subsequently, a total of 18,378 sequences were assigned to 25 KOG (clusters of orthologous groups for eukaryotic complete genomes) terms. Among the terms identified, the three highest represented categories were as follows: signal transduction mechanisms $(4,561)$; posttranslational modification, protein turnover, chaperones $(3,441)$; and general function prediction $(3,271)$ (Figure 1). All of the unigenes were queried in the GO database (Gene Ontology, an international standardized gene functional classification) to classify their predicted functions. From this analysis, 35,533 unigenes were grouped into three functional categories, "biological process", "cellular component", and "molecular function", with the subsets of sequences further divided into 33,18 , and 17 subcategories in these three groups, respectively. The largest subcategory in the "biological process" group was "metabolic process", which included $12.1 \%$ of the unigenes in the subcategory. In the "cellular component" and "molecular function" categories, "cell" and "binding activity" were the most abundant GO terms, representing $7.5 \%$ and $11.3 \%$ of each subcategory, respectively. In addition, there were high percentages of unigenes in the "cell part", "catalytic activity", and "cellular process" categories and only a few unigenes in the "receptor regulator activity", "locomotion", "cell junction", and "symplast" categories (Figure 2).

\section{Changes in gene expression under salt stress}

To investigate the molecular response to salt stress exposure, unigene transcript levels in the control and

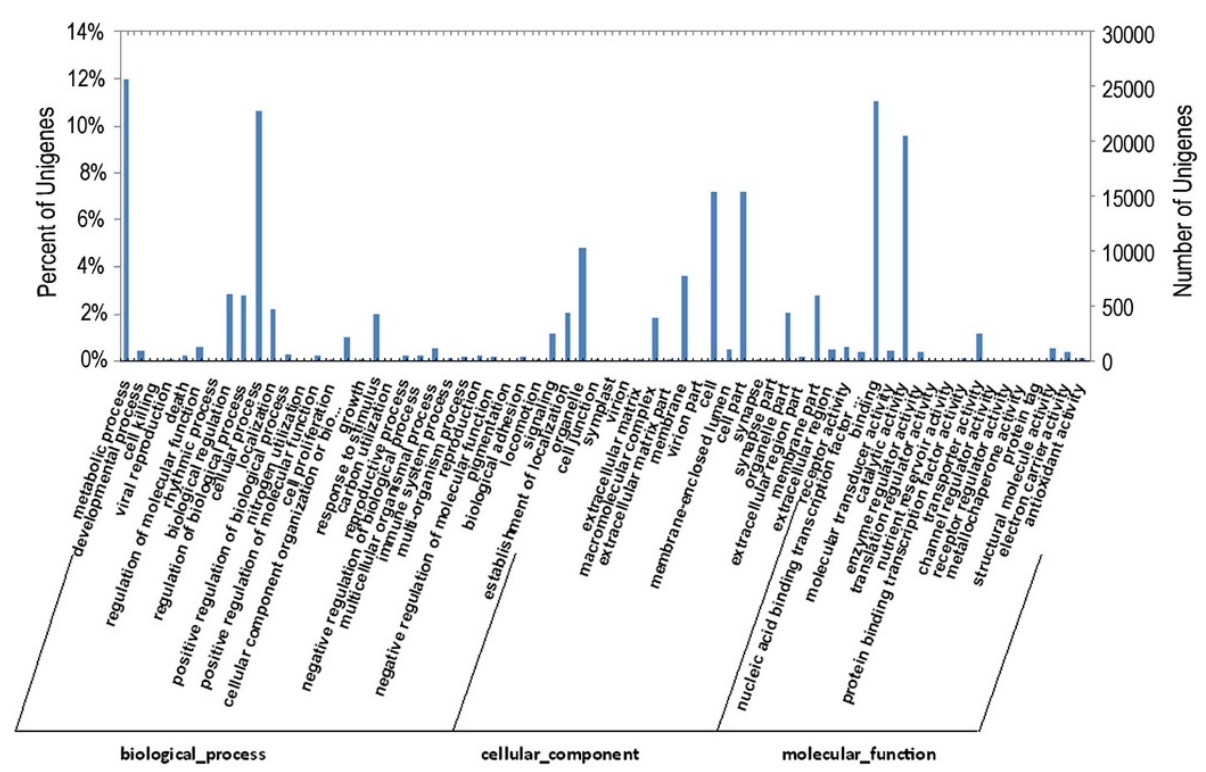

Figure 1 Diagram of the KOG (clusters of orthologous groups) classification. A total of 18,378 sequences were classified under 25 KOG categories. All of the unigenes were grouped into three major functional categories: biological process, cellular component, and molecular function. The right $y$-axis indicates the number of unigenes in a given category. The left $y$-axis indicates the percentage of a given category within the main category. 


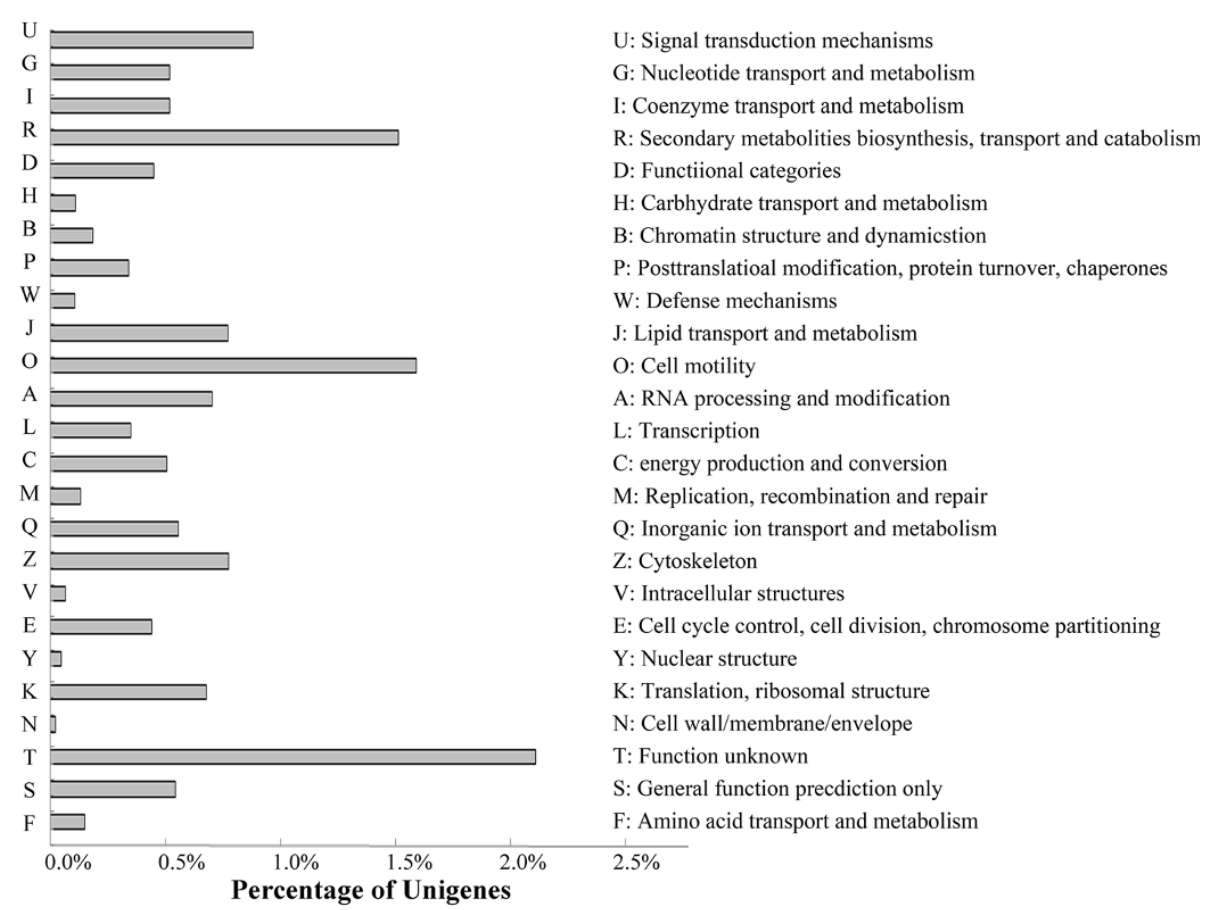

Figure 2 GO annotation of non-redundant unigenes. Good hits were aligned to the GO database, and 35,533 transcripts were assigned to at least one $\mathrm{GO}$ term.

salt stress treatments were calculated as RPKM (reads per kilobase per million reads), which eliminates the influence of gene length and sequencing discrepancy in calculating gene transcript levels and permits a direct comparison between treatments [19]. Based on the RPKM values observed, 60,127 differentially expressed unigenes were detected (Additional file 5: Table S5), with 38,123 and 22,005 up- and down-regulated genes, respectively. Salinity imposes a water deficit and ion stress, which have wideranging effects on the activity of plant cells, including inhibition of essential enzymes, cell membrane destabilization, a decrease in nutrient supply, and overproduction of reactive oxygen species (ROS) [15,21]. The extensive variation observed in the transcriptome (67.3\%) indicates complex transcriptional changes in K. caspica and comprehensive salt-stress influence on the cellular activity of $K$. caspica.

\section{Functional annotation of differentially expressed unigenes under salt stress}

To identify unigenes involved in metabolic or signal transduction pathways that were significantly enriched under salt stress, all of the differentially expressed sequences were queried in the KEGG database and compared to whole transcriptome data. Among the 60,127 differentially expressed transcripts (DETs), 13,848 genes were well annotated, while the remaining 46,239 genes had low sequence similarity to known sequences in the current database and therefore represent potentially novel salt-stress responsive genes. The potentially large number of unknown regulated genes suggested that factors involved in salt stress responses in Asteraceae may be distantly related to those identified in other genera. Pathway enrichment analysis revealed that many genes for which annotation data were available were directly or indirectly involved in the salt stress response, namely, primary metabolism, cellular processes, plant hormone signal transduction, plant-pathogen interaction, biosynthesis of secondary metabolism, and plant circadian rhythm (Figure 3 and Additional file 6: Table S6). These findings underscore the large scale re-coordination that occurs during short-term acclimation to salt stress exposure. Among the 4,023 DETs with pathway annotation, 172 DETs were found to be involved in the plant hormone signal transduction after salinity exposure, and 68 DETs were associated with MAPK signaling pathway (Table 1). Since very little information about the signaling cascades and the pathway of salinity sensing in Asteraceae is available, the presently identified sequences provide important clues for screening these putative salt stress responsive genes and their associated genes.

\section{Functional annotation of differentially expressed} unigenes involved in ABA-signaling under salt stress ABA plays a key role in a wide range of developmental processes and adaptive stress responses to environmental stimuli in plants [22]. Many studies have focused on the 


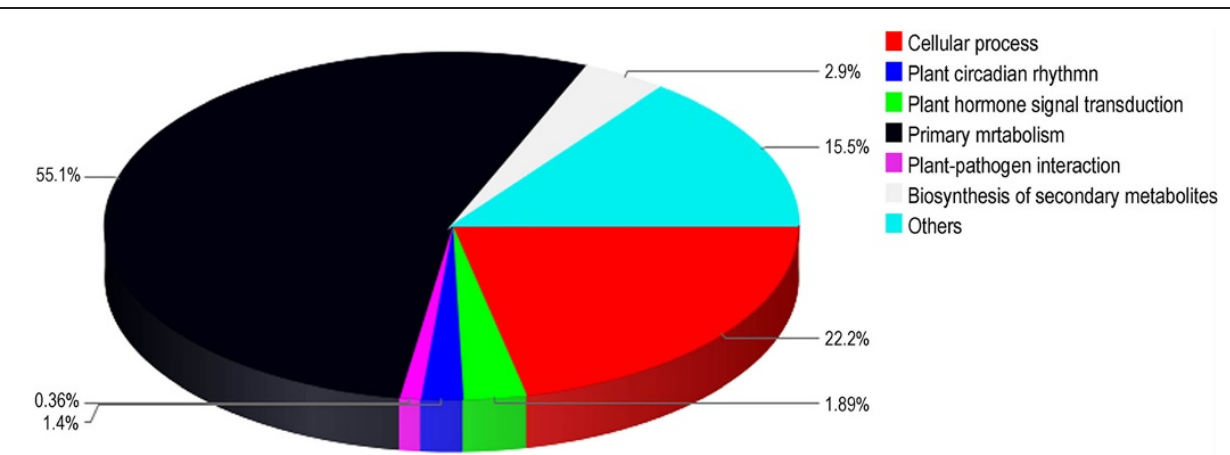

Figure 3 KEGG functional analysis of the differentially expressed unigenes.

cellular and molecular responses in plants to ABA, including ABA metabolism, transport, sensing, and signaling. We therefore focused on these physiological processes and highlighted potentially informative findings determined from the analysis.

\section{ABA metabolism}

ABA is synthesized from carotenoids. 9-cis-epoxycarotenoid dioxygenase (NCED) breaks down the 11, 12 double bond of 9-cis violaxanthin, which results in the formation of $\mathrm{C}_{15}$ xanthoxin within plastids. The subsequent steps of xanthoxin conversion to abscisic aldehyde are catalyzed by abscisic-aldehyde oxidase (AAO3) and xanthoxin dehydrogenase (ABA2) in the cytosol [23]. In the saltstressed $K$. caspica transcriptome, NCED (comp39466), AAO3 (comp43593), and ABA2 (comp714908) were all identified as up-regulated DETs. Consistent with this finding, carotenoid cleavage dioxygenase (CCD, comp40565), which yields another carotenoid-derived phytohormone from carotenoid and therefore competes with NCED for the same substrate, was down-regulated, further implicating enhanced ABA content in the responses of $K$. caspica to salt stress.

\section{ABA transport}

Although ABA is predominantly biosynthesized and metabolized in vascular tissues, it acts in the stomatal

\section{Table 1 Significantly enriched pathways of differentially} expressed unigenes

\begin{tabular}{lccc}
\hline Pathway category & Unigenes No & $\%$ & Qvalue \\
\hline Plant hormone signal transduction & 172 & 4.28 & $1.59 \mathrm{E}-14$ \\
MAPK signaling pathway & 68 & 1.69 & $1.45 \mathrm{E}-19$ \\
Calcium signaling pathway & 32 & 0.80 & $1.29 \mathrm{E}-14$ \\
Nitrogen metabolism & 29 & 0.72 & $2.61 \mathrm{E}-14$ \\
Circadian rhythm & 20 & 0.50 & $2.61 \mathrm{E}-14$ \\
Steroid hormone biosynthesis & 11 & 0.27 & $8.92 \mathrm{E}-12$ \\
\hline
\end{tabular}

Unigenes No. and \% indicate the number and the percentage of unigenes in each pathway from 4023 differentially expressed unigenes mapped to KEGG respectively. responses of distant guard cells [24-26]. In this way, ABA intercellular regulation and transport are critical for plant responsiveness to osmotic stress. It was recently reported that two G-type ATP-binding cassette $(\mathrm{ABC})$ transporter genes, AtABCG25 and AtABCG40, encode proteins responsible for ABA transport and response in Arabidopsis [27]. The ABC transporter is conserved in many model species from E. coli to humans and was reported to transport various metabolites and signaling molecules through the action of phytohormones in an ATP-dependent manner $[28,29]$. Several different types of ABC transporters (Table 2) were identified as up-regulated DEGs (differentially expressed genes) in $K$. caspica through BLAST homology searches, but only one unigene (comp306780) belonged to the G-type ABC subfamily. Nevertheless, this finding does not preclude the possibility that other non-G-type $A B C$ transporters or non-ABC transporters identified in $K$. caspica contribute to cell-to-cell ABA vesicular transport. In fact, two nitrate transporter 1/peptide transporters (NRT1/ PTR) family members involved in the transport of nitrogen (N) compounds were recently characterized as novel ABA transporters in Arabidopsis despite being wholly distinct from ABC transporter family members [23].

\section{ABA sensing and signaling}

In one proposed model of ABA signaling in Arabidopsis, PYR/RCAR/PYL (Pyrabactin Resistance/Regulatory Component of ABA Receptor/Pyrabactin Resistance 1-Like) family proteins, which act as $\mathrm{ABA}$ receptors, recognize and bind to group A PP2C (type $2 \mathrm{C}$ protein phosphatase) molecules in the presence of ABA. Subclass III SnRK2s (SNF1-related protein kinase 2) are then released from PP2C-dependent negative regulation, allowing the activated $\mathrm{SnRK} 2 \mathrm{~s}$ to phosphorylate downstream proteins such as ABA-responsive element (ABRE)-binding transcription factors (ABFs) [30-32]. Similar regulation of ABA signaling has been detected in other species such as wheat [30,33,34]. In K. caspica, orthologs of PYL (comp365319, comp38298), PP2C (comp6211), and SnRK2 (comp 30500, comp27671, comp38192) were up-regulated in the salt 
Table 2 Putative ATP-binding cassette $(A B C)$ transporter genes identified as up-regulated DEGs in $K$. caspica

\begin{tabular}{|c|c|c|c|c|c|}
\hline Unigene ID & Length (bp) & Subfamily & Best hit & E-value & Blast annotation/Organism \\
\hline comp465528 & 297 & A & Q99758 & $1 \mathrm{E}-63$ & ATP-binding cassette sub-family A member 3 [Homo sapiens] \\
\hline comp16398 & 388 & B & Q8LPT1 & $3 \mathrm{E}-54$ & ABC transporter B family member 6 [Arabidopsis thaliana] \\
\hline comp17267 & 328 & B & Q9ZR72 & $1 \mathrm{E}-59$ & ABC transporter B family member 1 [Arabidopsis thaliana] \\
\hline comp450357 & 451 & B & Q704E8 & $2 \mathrm{E}-61$ & ATP-binding cassette sub-family B member 7 [Rattus norvegicus] \\
\hline comp454701 & 282 & B & Q8LPK2 & $7 E-15$ & ABC transporter B family member 2 [Arabidopsis thaliana] \\
\hline comp577194 & 451 & B & Q9NRK6 & $3 \mathrm{E}-17$ & ATP-binding cassette sub-family B member 10 [Homo sapiens] \\
\hline comp20933 & 300 & C & Q9LZJ5 & $9 \mathrm{E}-47$ & ABC transporter C family member 14 [Arabidopsis thaliana] \\
\hline comp267971 & 705 & $\mathrm{D}$ & P48410 & $9 \mathrm{E}-125$ & ATP-binding cassette sub-family D member 2-like [Acyrthosiphon pisum] \\
\hline comp324163 & 542 & $\mathrm{D}$ & P48410 & $4 \mathrm{E}-40$ & ATP-binding cassette sub-family D member 1 [Mus musculus] \\
\hline comp352820 & 476 & $\mathrm{D}$ & Q9QY44 & $9 \mathrm{E}-45$ & ATP-binding cassette sub-family D member 2 [Rattus norvegicus] \\
\hline comp5337 & 228 & D & Q9UBJ2 & $3 \mathrm{E}-20$ & ATP-binding cassette sub-family D member 2 [Homo sapiens] \\
\hline comp481971 & 244 & $\mathrm{E}$ & P61222 & $5 \mathrm{E}-30$ & ATP-binding cassette sub-family E member 1 [Mus musculus] \\
\hline comp29933 & 2612 & $\mathrm{~F}$ & Q9UG63 & 0.0 & ATP-binding cassette sub-family F ember 2 [Tribolium castaneum] \\
\hline comp516400 & 351 & $\mathrm{~F}$ & Q8K268 & $2 \mathrm{E}-33$ & ATP-binding cassette sub-family F member 2 [Homo sapiens] \\
\hline comp306780 & 297 & G & Q9H172 & $1 \mathrm{E}-46$ & ATP-binding cassette sub-family G member 4 [Homo sapiens] \\
\hline
\end{tabular}

A

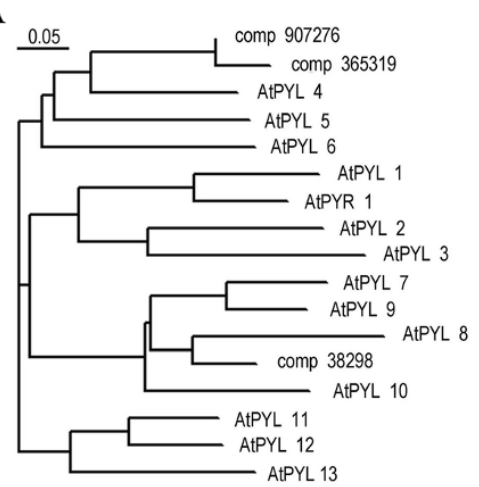

B

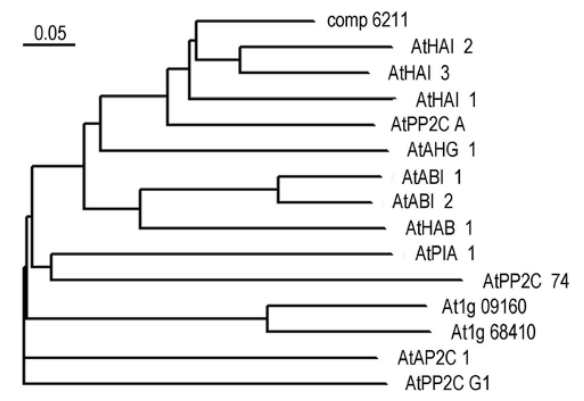

$\mathrm{C}$

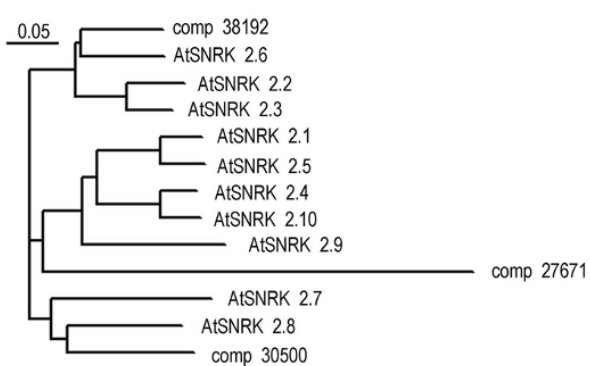

Figure 4 Sequence alignment and phylogenetic trees of (A) PYL, (B) PP2C, and (C) SnRK2 gene family members in Arabidopsis thaliana along with DEGs identified in $\boldsymbol{K}$. caspica. Sequence data referenced here can be found in the GenBank data libraries under the following accession numbers: PYL1 (AT5G46790), PYL2 (AT2G26040), PYL3 (AT1G73000), PYL4 (AT2G38310), PYL5 (AT5G05440), PYL6 (AT2G40330), PYL7 (AT4G01026), PYL8 (AT5G53160), PYL9 (AT1G01360), PYL10 (AT4G27920), PYL11 (AT5G45860), PYL12 (AT5G45870), PYL13 (AT4G18620), PYR1 (AT4G17870), SNRK2.1 (AT5G08590), SNRK2.2 (AT3G50500), SNRK2.3 (AT5G66880), SNRK2.4 (AT1G10940), SNRK2.5 (AT5G63650), SNRK2.6 (AT4G33950), SNRK2.7 (AT4G40010), SNRK2.8 (AT1G78290), SNRK2.9 (AT2G23030), SNRK2.10 (AT1G60940), PP2CA (AT3G1 1410), HAl1 (AT5G59220), AHG1(AT5G51760), PP2CG1(AT2G33700), AP2C1(AT2G30020), HAI2(AT1G07430), PIA1(AT2G20630), HAI3(AT2G29380), PP2C74(AT5G36250), AtHAB1 (AT1G72770), AtABI2(AT5G57050), and AtABI1(AT4G26080). 
stress sample (Figure 4), indicating that the regulation of ABA signaling is indeed conserved among plant species.

Based on phylogenetic analysis, the DEGs comp365319, comp38298, and comp907276 in K. caspica grouped together with AtPYL4-9 (Figure 4). Since AtPYL4, AtPYL5, AtPYL6, AtPYL8, and AtPYL9 have been found to inhibit PP2Cs even in the absence of ABA [35] and considering that the ectopic expression of PYL5 and PYL8 in Arabidopsis results in enhanced drought resistance [36,37], comp365319, comp38298, and comp907276 may function independently of ABA as positive regulators in the salinity response of $K$. caspica. Moreover, AtHAI1, AtHAI2, and AtHAI3 (Highly ABA-Induced PP2Cs) and the DEG comp6211 in $K$. caspica made up the clade A PP2Cs (Figure 4), which had the greatest effect on ABAindependent low water potential phenotypes and less of an effect on classical ABA sensitivity phenotypes [38]. Thus, comp6211 was associated with known clade A PP2Cs in ABA-independent salinity-associated signaling. Furthermore, physiological analyses illustrated that the
DEGs comp27671, comp30500, and comp38192 were classified into groups I, II, and III, respectively (Figure 4). Of these, only group III SnRKs are considered ABA-dependent kinases $[39,40]$. It nevertheless remains possible that many of the $K$. caspica DEGs may be involved in ABAindependent salinity-signaling cascades.

\section{Real-time PCR validation of differentially expressed unigenes}

To validate the transcriptome data of $K$. caspica under salt stress, five DEGs that were found to be up-regulated in K. caspica exposed to salt stress, namely, orthologs of SnRK2 (comp38192, comp 30500), PYL (comp38298, comp365319), and PP2C (comp6211), were selected for real-time PCR analysis using two-month-old $\mathrm{K}$. caspica seedlings treated with $300 \mathrm{mM} \mathrm{NaCl}$. As shown in Figure 5, all five genes exhibited enhanced expression at certain points during the treatment period, confirming the comparative transcriptome data for salt-stressed $\mathrm{K}$. caspica. Comp6211 and comp30500, which are predicted
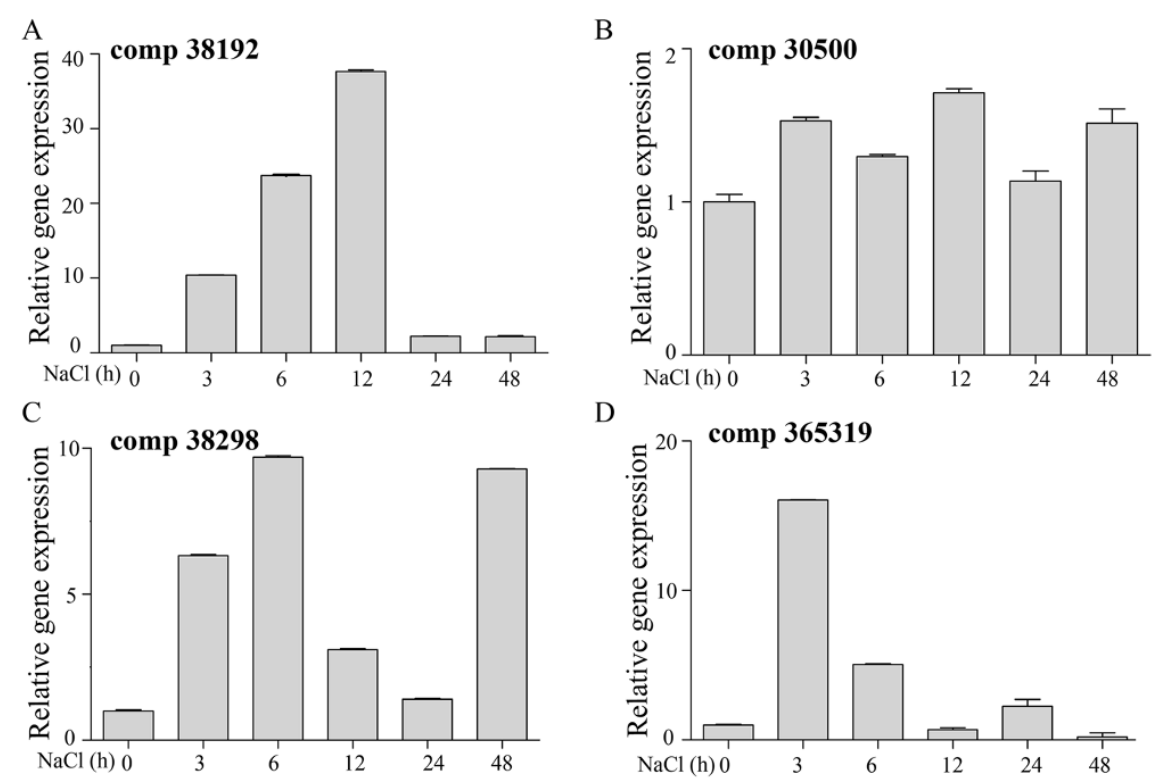

D
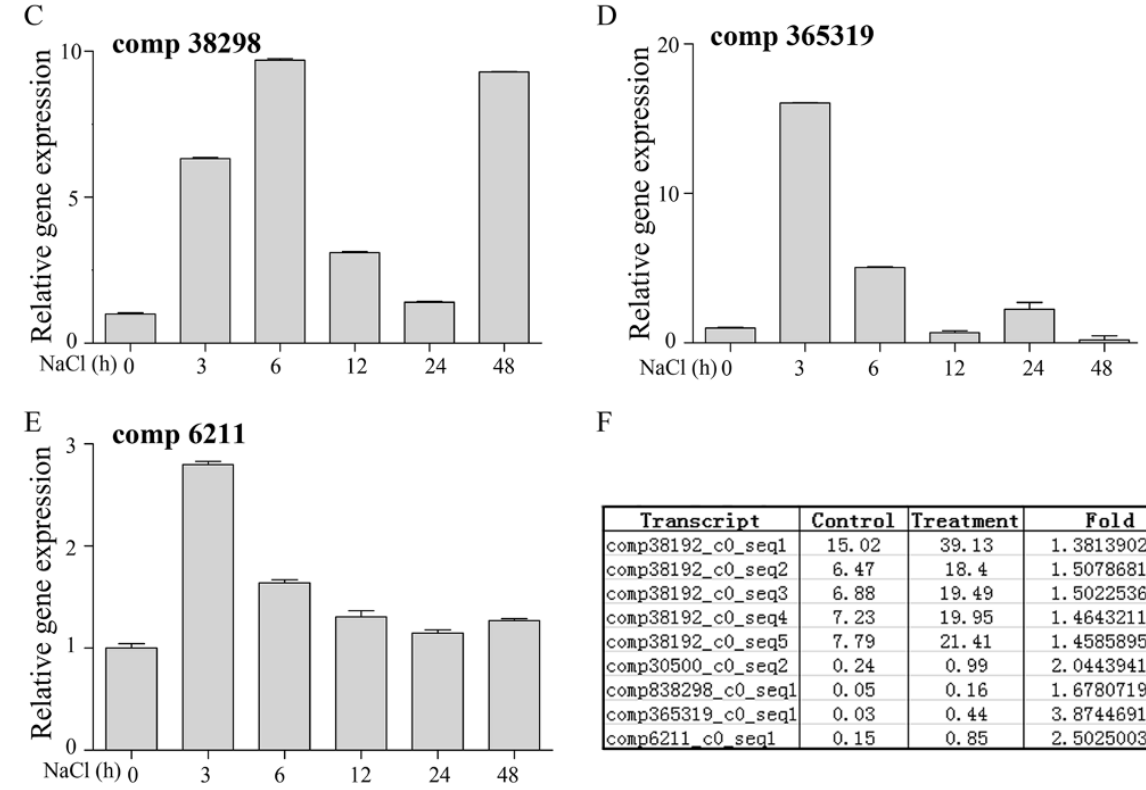

F

\begin{tabular}{|c|c|c|c|}
\hline Transcript & Control & Treatment & Fold \\
\hline comp38192_c0_seq1 & 15.02 & 39.13 & 1.381390297 \\
comp38192_c0_seq2 & 6.47 & 18.4 & 1.507868149 \\
comp38192_c0_seq3 & 6.88 & 19.49 & 1.502253621 \\
comp38192_c0_seq4 & 7.23 & 19.95 & 1.464321194 \\
comp38192_c0_seq5 & 7.79 & 21.41 & 1.458589562 \\
comp30500_c0_seq2 & 0.24 & 0.99 & 2.044394119 \\
comp838298_c0_seq1 & 0.05 & 0.16 & 1.678071905 \\
comp365319_c0_seq1 & 0.03 & 0.44 & 3.874469118 \\
comp6211_c0_seq1 & 0.15 & 0.85 & 2.502500341 \\
\hline
\end{tabular}

Figure 5 Real-time qRT-PCR analysis of selected genes from the RNA-seq dataset. Total RNA was extracted from K. caspica exposed to salt stress, and real-time PCR was used to validate the changes in gene expression of putative SnRK2 (comp38192, A; comp 30500, B), PYL

(comp38298, C; comp365319, D), and PP2C (comp6211, E). KcACTIN was used as the internal control. Error bars indicate standard deviations from three independent biological samples. And the fold change estimated by transcriptomic ananlysis were also illustrated in $\mathbf{F}$. 
to be ABA-independent signaling components, also changed over the course of the treatment period, implicating their functions in K. caspica responses to salinity. As ABA receptors, PYL (comp38298, C; comp365319, D)responded earlier in the presence of salinity stress, recognized and bind to group A PP2C (comp6211, E) molecules. Subclass III SnRK2s (comp38192, A; comp 30500, B), which responded to salinity stress at $12 \mathrm{~h}$, a little later than PYL (comp38298, C; comp365319, D) and PP2C (comp6211, E), are then activated, allowing the SnRK2s to phosphorylate downstream proteins. And the expression of the five genes was comparable with the fold change estimated by transcriptomic data illustating that the changes presented in real-time PCR are biologically significant. Taken together, these results validated the involvement of ABA in K. caspica subjected to salt stress. Actually, ABA is universal as stress-invlolved hormone in plant kimdom. Besides, the core components in ABA signaling have been obtained in rice, Selaginella moellendorffi, Physcomitrella patens, Ostreococcus tauri [41]. Although the PYR/PYL/RCAR-PP2CSnRK2 pathway model has been established [41], it is not clear whether this model can explain all ABA responses in plant kindom. It is necessary to determine whether these redundant variants are dependent on or independent of the core ABA pathway among different plants.

\section{Other DGEs involved in other hormone signaling}

Plants adapt to adverse environments by integrating growth and development to environmentally activate cues. Besides $A B A$, several DGE involved in other hormone signaling indicated multiple hormone crosstalks in K.caspica responses to salt stress. For example, GA integrates generic responses into abiotic stress tolerance via the DELLA proteins [42] (comp30323, comp47577) to regulate plant development. Auxin modulate plant development,especially root system architecture, to defense for salt stress by SAUR family protein (comp 31574, comp181105, etc.) and auxin responsive GH3 gene family (comp324952, comp9514, etc.) [43,44]; Brassinosteroid (BR) and ethylene, which respectively mediated by BR-signaling kinase (comp768925), ethyle-reponsive transcription factor (ERF1, comp20087; ERF2, comp382497), and EIN3-binding F-box protein (EBF1-2, comp768925), are also involved in strateges plants take to cope with unfavorable conditions[45,46]. Therefore, the response to salt stress in K.caspica is a comprehensive regulatory network in term of the plant hormones signaling cascade, and need further elucidated.

\section{Conclusion}

This study profiled the transcriptome of $K$. caspica under salt stress using Illumina RNA-seq technology to identify responsive genes and specific pathways involved in the salinity response of $K$. caspica. The transcriptome profile data provide a foundation for further investigation of the molecular basis underlying salt stress tolerance in this species. Several key genes involved in ABA metabolism, transport, sensing, and signaling functions were found to be induced by salt stress treatment. Thus, the transcriptome profiling approach and subsequent gene expression analysis in K. caspica provide important clues for the identification of functional genes and the contribution of the ABA signaling pathway to the salt tolerance of Asteraceae plants.

\section{Additional files}

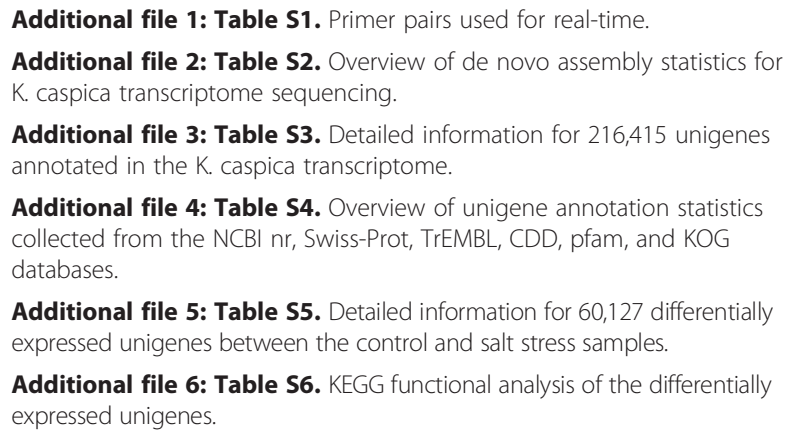

Additional file 3: Table S3. Detailed information for 216,415 unigenes annotated in the K. caspica transcriptome.

Additional file 4: Table S4. Overview of unigene annotation statistics collected from the NCBI nr, Swiss-Prot, TrEMBL, CDD, pfam, and KOG databases.

Additional file 5: Table S5. Detailed information for 60,127 differentially expressed unigenes between the control and salt stress samples.

Additional file 6: Table S6. KEGG functional analysis of the differentially expressed unigenes.

Competing interests

The authors declare that they have no competing interests.

\section{Authors' contributions}

ZX carried out the molecular genetic studies, participated in the design of the study, performed the data analysis, and drafted the manuscript. LM carried out the sequence alignment and performed the data analysis. CD participated in gene expression assay. ZF conceived of the study, and participated in its design and coordination and helped to draft the manuscript. All authors read and approved the final manuscript.

\section{Acknowledgements}

This work was supported by the National Natural Science Foundation of China (No. 30960035) and the 973 Pre-Research Project of the Ministry of Science and Technology, China (2012CB722204).

Received: 4 November 2013 Accepted: 11 December 2014 Published: 17 December 2014

\section{References}

1. Allakhverdiev SI, Sakamoto A, Nishiyama Y, Inaba M, Murata N: Ionic and osmotic effects of $\mathrm{NaCl}$-induced inactivation of photosystems I and II in Synechococcus sp. Plant Physiol 2000, 123:1047-1056.

2. Zhang YC, Rui TZ: Study on relationship between anatomical structure of leaves of Karelinia caspica (pall) less and ecological environment. J Ningxia Agric Coll 2003, 24(1):31-34.

3. Han $J$, Zhao KF: The structure, function, and salt-secreted mechanism of salt glands. J Shangdong Norm Univ (Nat Sci) 2001, 16(2):194-198.

4. Cheng CP: Modified saline soil-Kareliana caspica. Life Sci 1991, 4:6-7.

5. Wang Z, Gerstein M, Snyder M: RNA-Seq: a revolutionary tool for transcriptomics. Nat Rev Genet 2009, 10:57-63.

6. Nagalakshmi U, Wang Z, Waern K, Shou C, Raha D, Gerstein M, Snyder M: The transcriptional landscape of the yeast genome defined by RNA sequencing. Science 2008, 320:1344-1349.

7. Mortazavi A, Williams BA, McCue K, Schaeffer L, Wold B: Mapping and quantifying mammalian transcriptomes by RNA-Seq. Nat Methods 2008, 5:621-628. 
8. Pan $\mathrm{Q}$, Shai $\mathrm{O}$, Lee LJ, Frey J, Blencowe BJ: Deep surveying of alternative splicing complexity in the human transcriptome by high-throughput sequencing. Nat Genet 2008, 40:1413-1415.

9. Lister R, O' Malley RC, Tonti-Filippini J, Gregory BD, Berry CC, Millar AH, Ecker JR: Highly integrated single-base resolution maps of the epigenome in Arabidopsis. Cell 2008, 133:523-536.

10. Hillier LW, Reinke V, Green P, Hirst M, Marra MA, Waterston RH: Massively parallel sequencing of the polyadenylated transcriptome of $C$. elegans. Genome Res 2009, 19:657-666.

11. Zhang G, Guo G, Hu X, Zhang Y, Li Q, Li R, Zhuang R, Lu Z, He Z, Fang X, Chen L, Tian W, Tao Y, Kristiansen K, Zhang X, Li S, Yang H, Wang J, Wang J: Deep RNA sequencing at single base-pair resolution reveals high complexity of the rice transcriptome. Genome Res 2010, 20:646-654.

12. Zenoni S, Ferrarini A, Giacomelli E, Xumerle L, Fasoli M, Malerba G, Bellin D, Pezzotti M, Delledonne M: Characterization of transcriptional complexity during Berry development in Vitis vinifera using RNA-seq. Plant Physiol 2010, 152:1787-1795.

13. Léon-Kloosterziel K-M, Gil MA, Ruijs GJ, Jacobsen SE, Olszewski NE, Schwartz $\mathrm{SH}$, Zeevaart JAD, Koornneef M: Isolation and characterization of abscisic acid-deficient Arabidopsis mutants at two new loci. Plant J 1996, 10:655-661.

14. Chow B, McCourt P: Hormone signaling from a developmental context. J Exp Bot 2004, 55:247-251.

15. Zhu JK: Salt and drought stress signal transduction in plants. Annu Rev Plant Physiol 2002, 53:247-273.

16. Yao JT, Fu WD, Wang XL, Duan DL: Improved RNA isolation for Laminaria japonica Aresch (Laminariaceae Phaeophyta). J Appl Phycol 2008, 21:233-238.

17. Ye J, Fang L, Zheng H, Zhang Y, Chen J, Zhang Z, Wang J, Li S, Li R, Bolund L, Wang J: WEGO: a web tool for plotting GO annotations. Nucleic Acids Res 2006, 34:293-297.

18. Conesa A, Gotz S, Garcia-Gomez JM, Terol J, Talon M, Robles M: Blast2GO: a universal tool for annotation, visualization and analysis in functional genomics research. Bioinformatics 2005, 21:3674-3676

19. Marioni JC, Mason CE, Mane SM, Stephens M, Gilad Y: RNA-seq: an assessment of technical reproducibility and comparison with gene expression arrays. Genome Res 2008, 18:1509-1517.

20. Li H, Durbin R: Fast and accurate short read alignment with Burrows-Wheeler Transform. Bioinformatics 2009, 25:1754-1760.

21. Hasegawa PM, Bressan RA: Plant cellular and molecular responses to high salinity. Annu Rev Plant Physiol Plant Mol Biol 2000, 51:463-499.

22. Weiner JJ, Peterson FC, Volkman BF, Cutler SR: Structural and functional insights into core ABA signaling. Curr Opin Plant Biol 2010, 13(5):495-502.

23. Floss DS, Walter MH: Role of carotenoid cleavage dioxygenase 1 (CCD1) in apocarotenoid biogenesis revisited. Plant Signal Behav 2009, 4(3):172-175.

24. Cheng W, Endo A, Zhou L, Penney J, Chen H, Arroyo A, Leon P, Nambara E, Asami T, Seo M, Koshiba T, Sheen J: A unique short-chain dehydrogenase/ reductase in Arabidopsis glucose signaling and abscisic acid biosynthesis and functions. Plant Cell 2002, 14:2723-2743.

25. Koiwai H, Nakaminami K, Seo M, Mitsuhashi W, Toyomasu T, Koshiba T: Tissue-specifi clocalization of an abscisic acid biosynthetic enzyme, AAO3, in Arabidopsis. Plant Physiol 2004, 134:1697-1707.

26. Okamoto M, Tanaka Y, Abrams SR, Kamiya Y, Seki M, Nambara E: High humidity induces abscisic acid 8' -hydroxylase in stomata and vasculature to regulate local and systemic abscisic acid responses in Arabidopsis. Plant Physiol 2009, 149:825-834

27. Kuromori T, Miyaji T, Yabuuchi H, Shimizu H, Sugimoto E, Kamiya A, Moriyama Y, Shinozaki K: ABC transporter AtABCG25 is involved in abscisic acid transport and responses. Proc Natl Acad Sci 2010, 107:2361-2366.

28. Higgins CF: ABC transporters: from microorganisms to man. Annu Rev Cell Biol 1992, 8:67-113.

29. Rea PA: Plant ATP-binding cassette transporters. Annu Rev Plant Physiol 2007, 58:347-375

30. Kobayashi Y, Murata M, Minami H, Yamamoto S, Kagaya Y, Hobo T, Yamamoto A, Hattori T: Abscisic acid-activated SNRK2 protein kinases function in the gene-regulation pathway of $A B A$ signal transduction by phosphorylating ABA response element-binding factors. Plant J 2005 , 44:939-949.

31. Furihata T, Maruyama K, Fujita Y, Umezawa T, Yoshida R, Shinozaki K, Yamaguchi-Shinozaki K: Abscisic acid-dependent multisite phosphorylation regulates the activity of a transcription activator AREB1. Proc Natl Acad Sci 2006, 103:1988-1993.
32. Piskurewicz U, Jikumaru Y, Kinoshita N, Nambara E, Kamiya Y, Lopez-Molina L: The gibberellic acid signaling repressor RGL2 inhibits Arabidopsis seed germination by stimulating abscisic acid synthesis and ABI5 activity. Plant Cell 2008, 20:2729-2745.

33. Johnson RR, Wagner RL, Verhey SD, Walker-Simmons MK: The abscisic acid-responsive kinase PKABA1 interacts with a seed-specifi $c$ abscisic acid response element-binding factor, $\mathrm{TaABF}$, and phosphorylates TaABF peptide sequences. Plant Physiol 2002, 130:837-846.

34. Kagaya Y, Hobo T, Murata M, Ban A, Hattori T: Abscisic acid-induced transcription is mediated by phosphorylation of an abscisic acid response element binding factor, TRAB1. Plant Cell 2002, 14:3177-3189.

35. Santiago J, Rodrigues A, Saez A, Rubio S, Antoni R, Dupeux F, Park S, Márquez JA, Cutler SR, Rodriguez PL: Modulation of drought resistance by the abscisic acid receptor PYL5 through inhibition of clade A PP2Cs. Plant J 2009, 60:575-588.

36. Hao Q, Yin P, Li W, Wang L, Yan C, Lin Z, Wu J, Wang J, Yan SF, Yan N: The molecular basis of ABA-independent inhibition of PP2Cs by a subclass of PYL proteins. Mol Cell 2011, 42:662-672.

37. Saavedra X, Modrego A, Rodríguez D, González-García PM, Sanz L, Nicolás G, Lorenzo O: The nuclear interactor PYL8/RCAR3 of Fagus sylvatica FsPP2C1 is a positive regulator of abscisic acid signaling in seeds and stress. Plant Physiol 2010, 152:133-150.

38. Bhaskara $G B$, Nguyen $T$, Verslues PE: Unique drought resistance functions of the highly $\mathrm{ABA}$-induced clade $\mathrm{A}$ protein phosphatase 2Cs. Plant Physio 2012, 160(1):379-395

39. Kulik A, Wawer I, Krzywińska E, Bucholc M, Dobrowolska G: SnRK2 protein kinases-key regulators of plant response to abiotic stresses. OMICS 2011, 15(12):859-872.

40. Kim MJ, Park MJ, Seo PJ, Song JS, Kim HJ, Park C: Controlled nuclear import of the transcription factor NTL6 reveals a cytoplasmic role of SnRK2.8 in the drought-stress response. Biochem J 2012, 448(3):353-363.

41. Umezawa T, Nakashima K, Miyakawa T, Kuromori T, Tanokura M, Shinozaki K, Yamaguchi-Shinozaki K: Molecular basis of the core regulatory network in ABA responses: sensing, signaling and transport. Plant Cell Physio/ 2010, 51(11):1821-1839.

42. Achard P, Cheng H, De Grauwe L, Decat J, Schoutteten H, Moritz T, Van Der Straeten D, Peng J, Harberd NP: Integration of plant responses to environmentally activated phytohormonal signals. Science 2006, 311 (5757):91-94.

43. Wang Y, Li K, Li X: Auxin redistribution modulates plastic development of root system architecture under salt stress in Arabidopsis thaliana. J Plant Physiol 2009, 166:1637-1645.

44. Galvan-Ampudia CS, Testerink C: Salt stress signals shape the plant root. Curr Opin Plant Biol 2011, 14:296-302.

45. Gudesblat GE, Schneider-Pizoń J, Betti C, Mayerhofer J, Vanhoutte I, van Dongen W, Boeren S, Zhiponova M, de Vries S, Jonak C, Russinova E: SPEECHLESS integrates brassinosteroid and stomata signalling pathways. Nat Cell Biol 2012, 14:548-554.

46. Beguerisse-Dıaz M, Hernández-Gómez MC, Lizzul AM, Barahona M, Desikan R: Compound stress response in stomatal closure: a mathematical model of ABA and ethylene interaction in guard cells. BMC Syst Bio/ 2012, 6:146-161.

\section{doi:10.1186/1756-0500-7-927}

Cite this article as: Zhang et al:: Comparative transcriptome analysis of the Asteraceae halophyte Karelinia caspica under salt stress. BMC Research Notes 2014 7:927. 